\title{
Effective connectivity of cortical and subcortical regions during unification of sentence structure
}

\author{
Tineke M. Snijders a,b,c,d,e,*, Karl Magnus Petersson ${ }^{\text {a,b }}$, Peter Hagoort ${ }^{\text {a,b }}$ \\ a Radboud University Nijmegen, Donders Institute for Brain, Cognition and Behaviour, Centre for Cognitive Neuroimaging, The Netherlands \\ ${ }^{\mathrm{b}}$ Max Planck Institute for Psycholinguistics, Nijmegen, The Netherlands \\ c Rudolf Magnus Institute of Neuroscience, Department of Child and Adolescent Psychiatry, University Medical Centre Utrecht, Utrecht, The Netherlands \\ d Department of Experimental Psychology, Utrecht University, Utrecht, The Netherlands \\ e Department of Developmental Psychology, Utrecht University, Utrecht, The Netherlands
}

\section{A R T I C L E I N F O}

\section{Article history:}

Received 21 December 2009

Revised 26 April 2010

Accepted 13 May 2010

Available online 21 May 2010

\begin{abstract}
A B S T R A C T
In a recent fMRI study we showed that left posterior middle temporal gyrus (LpMTG) subserves the retrieval of a word's lexical-syntactic properties from the mental lexicon (long-term memory), while left posterior inferior frontal gyrus (LPIFG) is involved in unifying (on-line integration of) this information into a sentence structure (Snijders et al., 2009). In addition, the right IFG, right MTG, and the right striatum were involved in the unification process. Here we report results from a psychophysical interactions (PPI) analysis in which we investigated the effective connectivity between LPIFG and LpMTG during unification, and how the right hemisphere areas and the striatum are functionally connected to the unification network. LPIFG and LpMTG both showed enhanced connectivity during the unification process with a region slightly superior to our previously reported LpMTG. Right IFG better predicted right temporal activity when unification processes were more strongly engaged, just as LpIFG better predicted left temporal activity. Furthermore, the striatum showed enhanced coupling to LPIFG and LpMTG during unification. We conclude that bilateral inferior frontal and posterior temporal regions are functionally connected during sentence-level unification. Corticosubcortical connectivity patterns suggest cooperation between inferior frontal and striatal regions in performing unification operations on lexical-syntactic representations retrieved from LpMTG.
\end{abstract}

(c) 2010 Elsevier Inc. All rights reserved.

\section{Introduction}

In order to comprehend language we have to retrieve information about single words from long-term memory (mental lexicon) and combine ('unify') this information into representations that span multiple words (Marslen-Wilson, 1987; Levelt, 1992; Hagoort, 2005). Both memory and unification processes occur in parallel at the semantic (conceptual) and at the syntactic (structural) level (Jackendoff, 2002). Current syntactic theories tend to be lexicalist in nature: many properties relevant for structural integration are specified in the mental lexicon, rather than being computed by syntactic rules. Thus, structured syntactic properties of words (treelets; including, for example, word class and gender information) are retrieved from the lexicon, and the only remaining 'rule of grammar' is the combinatorial process of unification ('Unify Pieces' in Jackendoff (2002), somewhat similar to 'Merge' in Chomsky's

\footnotetext{
* Corresponding author. Utrecht University, Heidelberglaan 2, 3584 CS Utrecht, The Netherlands. Fax: + 31302534511.

E-mail address: T.M.Snijders@uu.nl (T.M. Snijders).
}

Minimalist Program (Chomsky, 1995)). Syntactic unification captures both sustained working memory processes during sentence comprehension (keeping lexical-syntactic information online) and the transient processes of selecting between alternatives and building of a phrase structure (manipulating this information) (Fiebach et al., 2005; Hagoort, 2005).

In a recent fMRI study, we showed that the left posterior middle temporal gyrus (LPMTG) subserves the retrieval of lexical-syntactic information from the mental lexicon, while the left posterior inferior frontal gyrus (LpIFG) is involved in combining this information online into a sentence structure (Snijders et al., 2009). The lexicalsyntactic information here specifies the possible structural environment of a lexical item (Vosse and Kempen, 2000). In the Snijders et al. study (2009), subjects read sentences and word sequences containing word-category (noun/verb) ambiguous words at critical positions. Regions contributing to the syntactic unification process should show enhanced activation for sentences compared to words, and increased activation for ambiguous compared to unambiguous conditions only when the target words are presented within a sentence, but not when presented in a random-word sequence. The LpIFG showed this predicted pattern, indicating that it is involved in syntactic unification. 
Presentation of a noun-verb ambiguous word in a neutral context triggers the retrieval of both the noun and the verb version of the ambiguous word (Seidenberg et al., 1982; Duffy et al., 1988). Thus, regions subserving the retrieval of lexical-syntactic information from memory should show more activation for ambiguous than unambiguous conditions (i.e., main effect over both sentences and randomword sequences). This pattern was observed in the LpMTG, signaling its involvement in the retrieval process. The pattern of results suggested a dynamic interplay between these two regions in the unification process (Snijders et al., 2009). Additionally, we found activations in right inferior frontal gyrus (RIFG) and right midposterior middle temporal gyrus (RpMTG) for ambiguous compared to unambiguous conditions when presented in sentences but not when presented in word sequences. We also found an effect of ambiguity in the striatum (part of the basal ganglia, BG) in both word sequence and sentence contexts (for further details, see Snijders et al. (2009)).

A number of studies, predominantly outside the language domain, have shown that the sustained activation of representations in posterior cortices is under the dynamic top-down control of frontal cortex (Tomita et al., 1999; Fuster, 2001; Miller and Cohen, 2001; Curtis and D'Esposito, 2003; Miller and D'Esposito, 2005; Fiebach et al., 2006; Fuster, 2008). Furthermore, these representations can be manipulated, flexibly updated, and integrated over time in the frontal cortex (Thompson-Schill et al., 1997; Fuster, 2008). Syntactic unification might, therefore, be the result of LpIFG interacting with representational brain regions in the posterior temporal lobe (Snijders et al., 2009). This hypothesis was not directly tested in our previous fMRI study where we only examined the pattern of activation, rather than directly testing whether LPIFG and LPMTG were functionally connected during syntactic unification. If lexicalsyntactic representations in LPMTG are modulated by frontal topdown processes, then we should observe evidence of "crosstalk" between LPIFG and LPMTG during language processing - evidence of effective connectivity - rather than mere co-activation of the two regions of interest. The aim of the current study, therefore, was to explicitly examine the connectivity between LPIFG and LpMTG during unification processes by testing for psychophysiological interactions (PPI) (Friston et al., 1997). We tested which brain areas showed enhanced coupling with LPIFG and LPMTG during sentence-level unification. We hypothesized that effective connectivity between LPIFG and LPMTG would be larger in ambiguous than in unambiguous sentences, and that the ambiguity effect would be absent for word sequences.

Classically, language processing has been localized in the left frontal and temporal brain regions. The role of right hemisphere ( $\mathrm{RH})$ regions and the basal ganglia in language processing is still a matter of debate (RH e.g.: Faust and Chiarello, 1998; Kircher et al., 2001; Bookheimer, 2002; Jung-Beeman, 2005); BG e.g.: (Copland et al., 2000; Lieberman, 2001; Friederici et al., 2003; Frisch et al., 2003; Ullman, 2004; Crosson et al., 2007). In the present study we also examined if the RIFG, RpMTG, and the striatum (regions showing an ambiguity effect in our previous study, see above) are functionally connected to the unification network. We were interested in whether 1 ) the right hemispheric regions show connectivity patterns similar to their left hemispheric counterparts, 2) the striatum shows enhanced connectivity to LpIFG and/or LpMTG during ambiguity processing, and 3 ) if this enhanced connectivity differs in a sentence versus a word-list context.

Thus, the goal of the current study was to identify how combinatorial processes in language modulate the connectivity between left frontal and temporal regions in the brain, and how the $\mathrm{RH}$ and the striatum are functionally connected to the unification network. More specifically, we investigated how word-category ambiguity in a sentence versus a word context alters the connectivity in cortical and subcortical networks.

\section{Materials and methods}

\section{Participants}

Twenty-eight right-handed healthy volunteers (14 females, mean age 23, range 18-35) participated in the experiment. All participants were highly educated native Dutch speakers with no history of neurological illness or head injury. Subjects were paid for their participation. Six additional subjects were scanned but excluded from analysis because of excessive movement in the MR-scanner (2 subjects) or poor task performance (4 subjects, see below). The study was approved by the local ethics committee and all participants gave written informed consent prior to the experiment in accordance with the Declaration of Helsinki.

\section{Stimulus material}

The stimuli consisted of 68 (Dutch) sentences (S) and 68 matched scrambled sequences of Dutch words $(\mathrm{W})$. Both the sentences and the word sequences contained a critical word that was either word-class (noun/verb) ambiguous (A) or unambiguous (U). The critical word in the sentences was disambiguated by the continuation of the sentence into either a noun $(\mathrm{n})$ or a verb (v) reading. In total, this resulted in eight possible conditions: SAn, SAv, SUn, SUv, WAn, WAv, WUn and WUv (see Table 1 for examples).

The ambiguous words were equibiased, that is, there was no strong preference for the noun over the verb interpretation or vice versa. This was brought out both by lexical frequencies as occurring in Dutch lexical databases (Baayen et al., 1993; Beek et al., 2001), as well as by a pre-test, on a different group of participants, where subjects had to complete sentence fragments ending with the ambiguity (e.g. "Zodra jullie bewijzen..."). The ambiguous sentences were constructed such that both categories of the critical word fitted syntactically as well as semantically with the initial part of the sentences (up to and including the critical word); the sentences were disambiguated by the subsequent part of the sentence (after the ambiguous word; see Table 1 for an example). Word sequences were constructed from the sentences by substituting every word (except the critical word) by a different word, matched for length, frequency, and word category, and subsequently scrambling the order of the words in the sequence (except the critical word). For a full description of experimental materials, see Snijders et al. (2009).

\section{Procedure}

Stimuli were presented using the serial visual presentation method (i.e. word by word in the middle of the screen) using the Presentation software (Version 9.13, www.neuro-bs.com). Every word remained on the screen for $300 \mathrm{~ms}$, with a $200 \mathrm{~ms}$ interword-interval. Between the sequences of words (sentences or random), a visual fixation cross was presented for 5-8 s (low-level baseline). The participants were instructed to read each sentence/ sequence carefully and attentively, and were told that after the experiment some questions concerning the experiment would have to be answered. The participants' task was spotting the consonantstrings (e.g., $c d s n l$ ), that were presented in 36 of the fillers (18 sentences and 18 sequences). This simple control task was added to check whether subjects were paying attention. Subjects were defined as poor task performers if they made more than five errors (missing hits and false alarms) on the task, suggesting that these subjects did not pay enough attention to the stimuli.

Every subject saw 68 sentences and 68 word sequences (ambiguous/unambiguous; in noun/verb version), intermingled with 56 fillers (28 sentences and 28 sequences). No subject encountered the same critical word or context sentence/sequence more than once. Stimuli were presented in mini-blocks of three to four sentences or 
Table 1

Example of the experimental materials, with the critical word bewijzen (evidence/to prove).

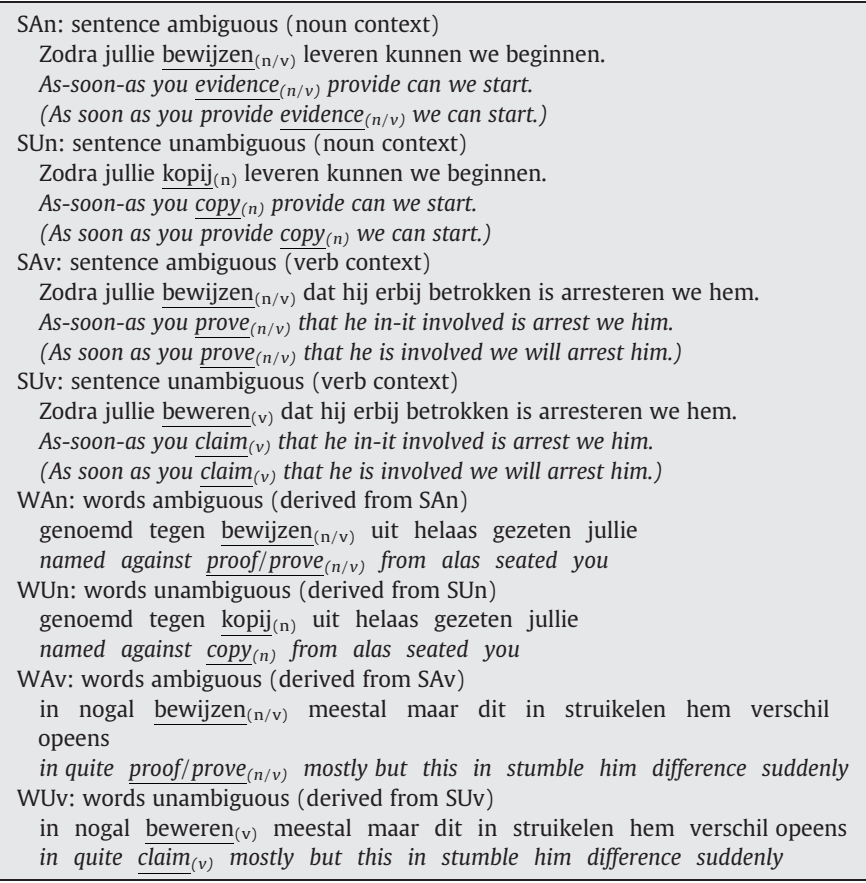

Critical words are underlined; the English translations are italicized.

word sequences. All mini-blocks were shorter than $40 \mathrm{~s}$. Before each block the label "Zinnen:" ("Sentences:") or "Woorden:" ("Words:") appeared on the screen (for $1.5 \mathrm{~s}$ ) to indicate the condition of the following mini-block, which started after a fixation cross of 1-3 s. We expected the labels to encourage ("Sentences") or discourage ("Words") attempts to syntactically/semantically integrate the stimulus items in the upcoming mini-block. The ambiguous/unambiguous and verb/noun conditions were intertwined within the miniblocks in a pseudo-randomised presentation order.

\section{fMRI data acquisition}

During the sentence/sequence presentation we acquired $\mathrm{T}_{2}^{*}$ weighted EPI-BOLD fMRI data with a SIEMENS Trio $3 \mathrm{~T}$ MR-scanner in one session, using an ascending slice acquisition sequence ( $\sim 1190 \mathrm{EPI}$ volumes, volume $\mathrm{TR}=2 \mathrm{~s}, \mathrm{TE}=35 \mathrm{~ms}, 90^{\circ}$ flip-angle, 29 slices, slicematrix size $=64 \times 64$, slice thickness $=3 \mathrm{~mm}$, slice gap $=.5 \mathrm{~mm}$, FOV $=224 \mathrm{~mm}$, voxel size $=3.5 \times 3.5 \times 3.0 \mathrm{~mm}$ ). At the end of the scanning session, a structural MR image volume was acquired for which a high-resolution T1-weighted 3D MPRAGE sequence was used ( $\mathrm{TE}=3.93 \mathrm{~ms}, 8^{\circ}$ flip-angle, 192 sagittal slices, slice thickness $=1.0 \mathrm{~mm}$, voxel size $=1 \times 1 \times 1 \mathrm{~mm}$ ).

\section{Data analysis}

To enable comparisons with the published conventional analysis of this study (Snijders et al., 2009) we preprocessed the data in the identical manner. See Snijders et al. (2009) for the results of the conventional subtraction analysis. The group results of this analysis were used for choosing the regions of interest (ROIs) for the connectivity analysis.

Image preprocessing and statistical analysis were performed using Statistical Parametric Mapping (SPM2; www.fil.ion.ucl.ac.uk/spm). The first five image volumes were discarded in order to avoid transient non-saturation effects. The functional EPI-BOLD images were realigned, slice-time corrected, and the subject-mean functional MR images were co-registered with the corresponding structural MR images using mutual information optimization. Subsequently, functional images were normalized to a Montreal Neurological Institute (MNI)-aligned echo planar imaging template (based on 28 male brains acquired on the Siemens Trio at the Donders Centre) and resampled to an isotropic voxel size of $2 \mathrm{~mm}^{3}$. Finally, the normalized images were spatially filtered by convolving the functional images with an isotropic 3D Gaussian kernel (10 mm full width at half maximum).

The fMRI data were proportionally scaled to account for various global effects, and analyzed statistically using the general linear model and statistical parametric mapping, using a mixed-design procedure (Friston et al., 2007). At the first-level, single-subject fixed effect analyses were conducted. The linear model included mini-block regressors to model the sentence/sequence presentation from the onset of the critical word to the offset of the sentence/sequence-final word. The beginnings of sentences/sequences and filler items were modeled together as a regressor of no interest (other words, OW), and the presentation of the fixation cross (FIX) was modeled as explicit baseline. We temporally convolved the explanatory variables with the canonical hemodynamic response function provided by SPM2. We included the realignment parameters for movement artifact correction and a temporal high-pass filter (cut-off $128 \mathrm{~s}$ ) to account for various low-frequency effects as effects of no interest. Temporal autocorrelation was modeled as a first-order plus white noise autoregressive process.

For the second-level whole brain subtraction analysis, we generated single-subject contrast images for the SAn, WAn, SUn, WUn, SAv, WAv, SUv, and WUv items relative to the baseline FIX, and used these in a oneway random effects repeated measures ANOVA (including the factors: condition [8] and subject [28]). SPM[T] volumes were generated for the effect of grammaticality (i.e., sentences $>$ words) and the effect of ambiguity (ambiguous > unambiguous). Additionally, SPM[T] volumes were created for the effect of ambiguity within the sentences or word sequences context separately ( $\mathrm{SA}>\mathrm{SU}$ and $\mathrm{WA}>\mathrm{WU}$ ). For the results of this subtraction analysis, see Snijders et al. (2009).

\section{Regions of interest (ROIs)}

Regions of interest were chosen based on involvement in the syntactic unification process (Snijders et al., 2009). Seven regions of interest (ROIs) were specified for each individual: the left posterior inferior frontal gyrus (LPIFG, BA 44/6/9), the left anterior inferior frontal gyrus (LaIFG, BA 45/47), the left posterior middle temporal gyrus (LpMTGi, BA 37/20/21), the right inferior frontal gyrus (RIFG, BA 45 ), the right mid-posterior middle temporal gyrus (RpMTG, BA 20), the right striatum (Rstriatum), and the left striatum (Lstriatum). Fig. 1 illustrates the spatial location of these ROIs on a model brain. The ROIs were defined as $8-\mathrm{mm}$ radius spheres with the origin at specific coordinates based on the group-analysis results of Snijders et al. (2009). For LpIFG, RIFG, and RpMTG peak coordinates were taken from the sentence-ambiguous $>$ sentence-unambiguous $(S A>S U)$ contrast. For both the $\mathrm{SA}>\mathrm{SU}$ contrast and the main effect of ambiguity $(A>U)$ the peak voxel in LpMTG was at $[-52-50-8]$, which was taken as ROI centre for LPMTG. As this ROI was located on the border of the inferior temporal sulcus, we will refer to it as LpMTGi from now on. The right striatum showed a main effect of ambiguity (Snijders et al., 2009), we used the peak voxel ([24 0 8 $]$ ) for ROI definition. Additionally the left hemispheric counterpart of the right striatum ROI was added as ROI (Lstriatum). Furthermore we added a more anterior part of the LIFG as a control ROI (LaIFG, [-42 266]). A meta-analysis (Bookheimer, 2002) suggests that activations related to semantic processing in LIFG are centered around these coordinates (Petersson et al., 2004). All ROIs are displayed in Fig. 1; see the figure caption for MNI coordinates.

\section{Psychophysiological interactions analysis}

In the current study we explored whether the ROIs show differential coupling with other brain regions depending on the 


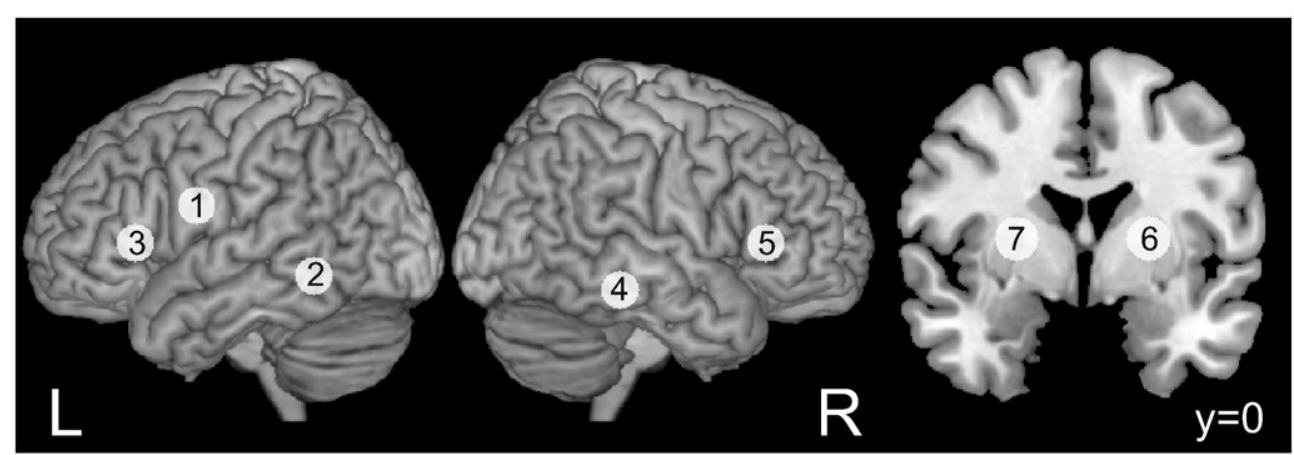

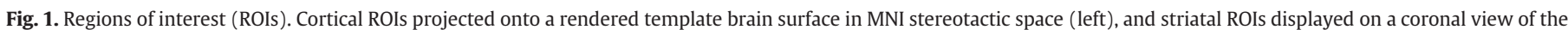

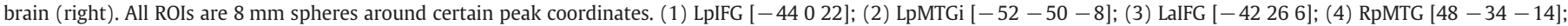
(5) RIFG [46 28 6]; (6) Rstriatum [24 0 8]; (7) Lstriatum [-24 0 8].

experimental conditions, using the psychophysiological interactions (PPI) described by Friston et al. (1997). The statistical model testing for psychophysiological interactions is a simple regression model of effective connectivity (Friston, 2002). Friston et al. define the contribution of a seed region to another region as the degree to which the activity in the second region can be predicted on the basis of activity of the first. A psychophysiological interaction reflects the change in this contribution depending on an experimental manipulation (Friston et al., 1997; Friston, 2002). Thus, a psychophysiological interaction expresses which brain regions (on a voxel-by-voxel basis) show an enhanced coupling (as evidenced by a steeper regression slope) with a region of interest (seed region) during one experimental condition compared to another condition (Friston et al., 1997). There are two possible interpretations of a PPI (see Friston et al., 1997): a condition change (in our case: ambiguity) modulates the degree to which activity in one region can be predicted on the basis of activity in the seed region, or, the seed region modulates the response of another region to the psychological factor (ambiguity).

For every ROI, two PPI analyses were performed: one looking for enhanced coupling of the seed region with other regions in the brain for sentence-ambiguous compared to sentence-unambiguous conditions (SA $>\mathrm{SU}$ ), and one looking for enhanced coupling of the seed region with other regions in the brain for word-ambiguous compared to word-unambiguous conditions (WA $>W U$ ).

For each subject and for each ROI, the physiological activity of the seed regions was summarized as the first eigenvariates of the time series of all active voxels within an $8 \mathrm{~mm}$ radius sphere centered on the most significant voxel within the ROI. Significance of voxels was based on the following contrasts: for LIFG and RIFG we used the sentences $>$ words contrast to identify active voxels, while for LpMTGi, RpMTG, and the striatum the ambiguous $>$ unambiguous contrast was used (both $p<.05$ uncorrected). When there were less than 5 voxels in the ROI that met the above criteria, the statistical threshold was eased on an individual subject basis (see Supplementary Table 1). To estimate underlying neuronal activity the physiological activity of the seed region was deconvolved (Gitelman et al., 2003).

The PPI regressor was obtained by multiplying the estimated neuronal activity from the seed region with a vector coding for effects of ambiguity within word lists or sentences (SA $>$ SU: 1 for ambiguoussentence condition, -1 for unambiguous-sentence condition; WA $>$ WU: 1 for ambiguous-word condition, -1 for unambiguousword condition; see above). Then, a whole brain analysis (singlesubject level) was performed using the general linear model in SPM2, using as predictor variables the PPI regressor, the experimental contrast $(\mathrm{SA}>\mathrm{SU}$ or $\mathrm{WA}>\mathrm{WU})$, and the estimated neuronal activity from the seed region. We temporally (re)convolved the explanatory variables with the canonical hemodynamic response function provided by SPM2. We included the realignment parameters for movement artifact correction and a temporal high-pass filter (cut- off $128 \mathrm{~s}$ ) to account for various low-frequency effects as effects of no interest. Temporal autocorrelation was modeled as a first-order plus white noise autoregressive process. For each ROI, participant specific PPI models were run, and contrast images were generated for the PPI regressor. The identified regions have greater connectivity with the seed region for ambiguous than unambiguous conditions.

Finally, for each seed region, individual PPI contrast images were entered into a one-sample $t$-test at the second (group) level. Whole brain family-wise error correction for multiple comparisons was applied by combining a significance level of $p<.005$, uncorrected at the voxel-level, with a cluster extent threshold of $p<.05$ corrected for multiple comparisons (Forman et al., 1995; Friston et al., 1996).

\section{Anatomical inference}

All local maxima are reported as MNI coordinates (Evans et al., 1993). Relevant anatomical landmarks were identified and Brodmann areas were defined using the Atlas of the Human Brain (Mai et al., 2004) and MRIcro (Rorden and Brett, 2000) using the AAL template (Tzourio-Mazoyer et al., 2002) and the Talairach Daemon (Lancaster et al., 2000).

\section{Results}

\section{Left hemisphere}

\section{Seed region: LPIFG}

We hypothesized that the LpIFG would modulate the activity in the LPMTG in response to the unification process. Indeed, LPIFG showed an enhanced coupling during sentence-ambiguous compared to sentence-unambiguous conditions with a region in LPMTG, bordering the superior temporal sulcus (LpMTGs; see Fig. 2A and Table 2). The activated region in LpMTGs was slightly superior to our region of interest in LPMTG (our ROI, LPMTGi, being located on the border of the inferior temporal sulcus). See Fig. 3A for a comparison of the two regions. No regions showed larger connectivity with LpIFG for word-ambiguous compared to word-unambiguous conditions. Thus, only in a sentence context the LPIFG is modulating activity in LPMTGs more for ambiguous than unambiguous items.

\section{Seed region: $L a I F G$}

To explore whether other regions within LIFG are in a similar way modulating posterior regions during unification, we included another region of the LIFG, which was located more anterior than LpIFG, to serve as a control ROI (LaIFG, see Materials and methods). LaIFG showed no enhanced coupling with other brain regions for sentenceambiguous compared to sentence-unambiguous conditions, nor for word-ambiguous compared to word-unambiguous conditions (see Table 2). Thus, the enhanced connectivity between LpIFG and LPMTGs is specific to the posterior part of LIFG. 


\section{A Seed LpIFG; PPI SA>SU}

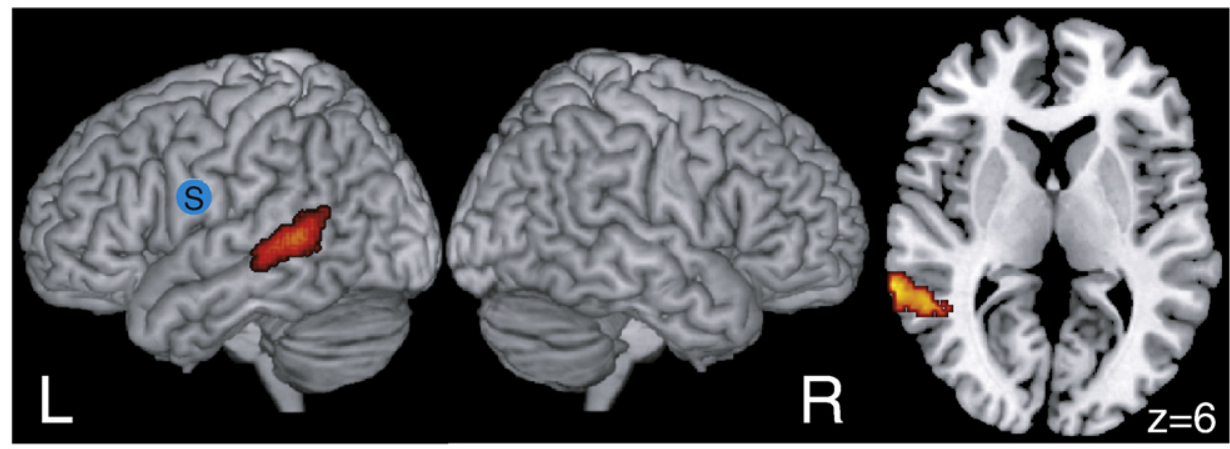

B Seed LPMTGi; PPI SA>SU; PPI WA>WU

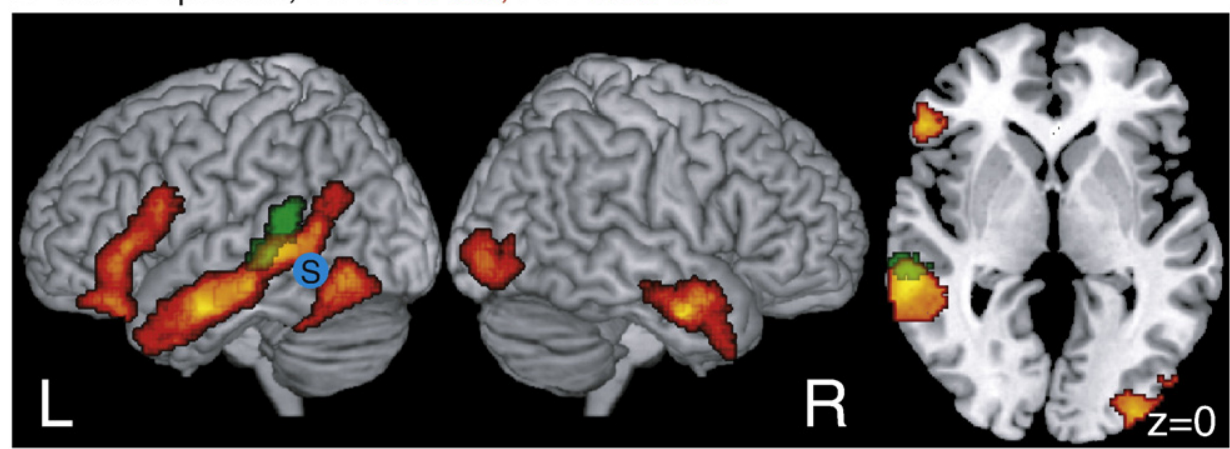

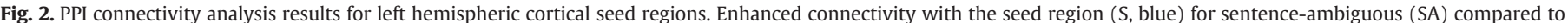

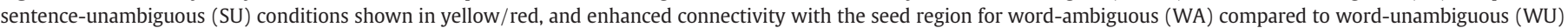

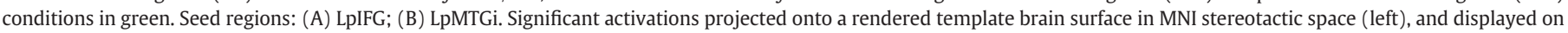
an axial view of the brain (right). The left side of the axial image corresponds to the left side of the brain. Activations shown at voxel-level $P_{\text {uncorr }}<.005$, cluster-level $P_{\text {FwE }}<.05$.

\section{Seed region: $L p M T G i$}

LPMTGi showed more connectivity for sentence-ambiguous than for sentence-unambiguous conditions with left inferior frontal gyrus (LIFG), as well as with left middle temporal gyrus (LMTG, superior to ROI), right anterior middle temporal gyrus (RaMTG), left posterior inferior temporal gyrus (LPITG) and right occipital gyrus (ROcG; see Fig. 2B and Table 2). The activated region in LpITG coincides with the so-called visual word form area (VWFA, see e.g. McCandliss et al.,
2003). For word-ambiguous compared to word-unambiguous conditions LpMTGi showed enhanced coupling with a region in left midMTG/posterior STG (see Fig. 2B (green) and Table 2).

Thus, for ambiguous sentences there is more connectivity between LPMTG and LIFG than for unambiguous sentences. The enhanced connectivity between LIFG and LPMTG as a result of wordcategory ambiguity (or ambiguity resolution) was only evident in the sentence, but not in the word sequence, condition. Both LPIFG
A

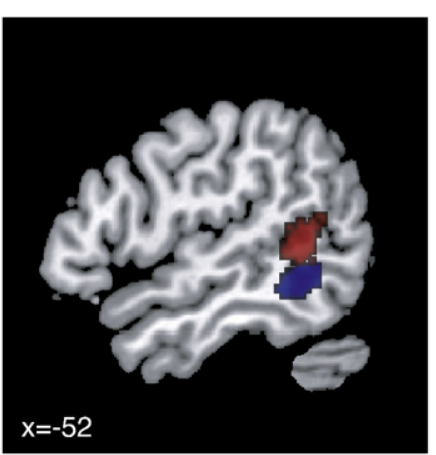

LpMTGs
B

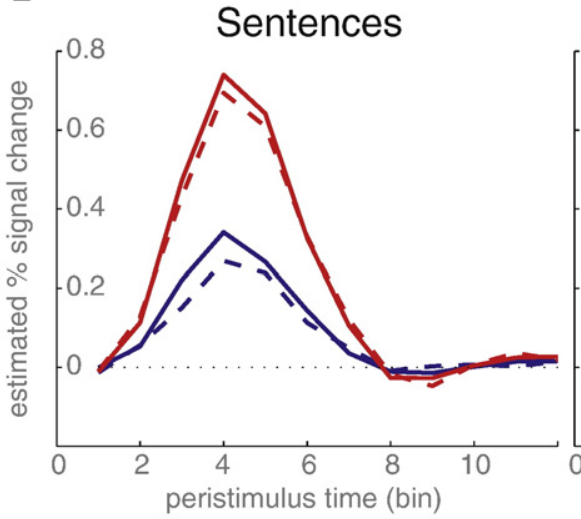

- Ambiguous

\section{Word sequences}

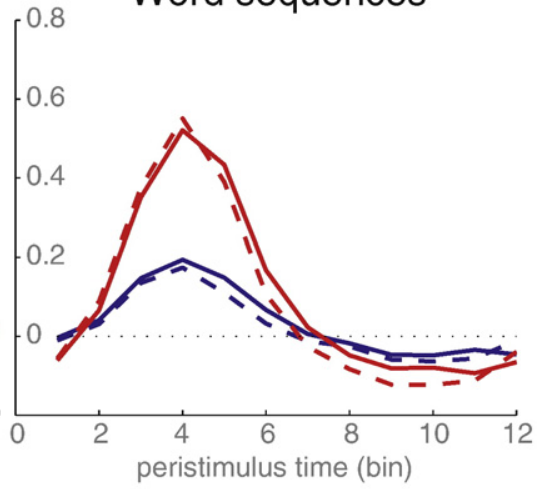

-- - Unambiguous

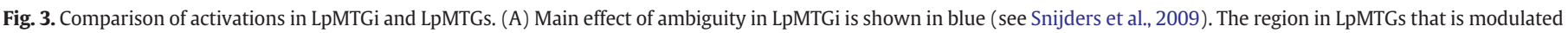

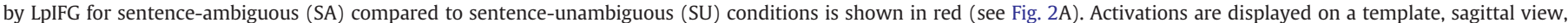

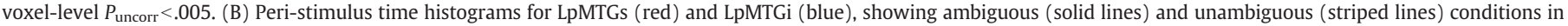

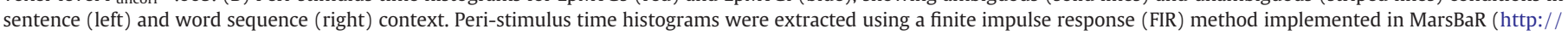
marsbar.sourceforge.net/). Every time bin consists of 1 TR (2s). 
Table 2

PPI connectivity results for left hemispheric cortical seed regions (LPIFG, LpMTGi, and LaIFG).

\begin{tabular}{|c|c|c|c|c|c|c|c|c|c|}
\hline Seed & PPI & Region & BA & $\begin{array}{l}\text { Cluster } \\
\text { size }\end{array}$ & $\begin{array}{l}\text { Cluster } p \\
\text { (corrected) }\end{array}$ & $\begin{array}{l}\text { Voxel } \\
T^{27} \text { value }\end{array}$ & $x$ & $y$ & $z$ \\
\hline LpIFG & $\mathrm{SA}>\mathrm{SU}$ & L posterior MTG/STS & & 443 & .002 & & & & \\
\hline$\left[\begin{array}{lll}-4 & 0 & 22\end{array}\right]$ & & L post-MTG/STS & 22 & & & 4.76 & -68 & -40 & 6 \\
\hline \multirow[t]{3}{*}{ BA $44 / 6 / 9$} & & L post-MTG & 21 & & & 3.91 & -54 & -50 & 6 \\
\hline & & L mid-MTG & 21 & & & 3.73 & -68 & -28 & 0 \\
\hline & $\mathrm{WA}>\mathrm{WU}$ & No significant clusters & & & & & & & \\
\hline LpMTGi & $\mathrm{SA}>\mathrm{SU}$ & LMTG & & 2383 & $<.001$ & & & & \\
\hline$[-52-50-8]$ & & L mid-M/ITG & $20 / 21$ & & & 6.21 & -56 & -24 & -14 \\
\hline \multirow[t]{22}{*}{ BA $37 / 20 / 21$} & & L ant-MTG & 21 & & & 5.32 & -56 & -2 & -24 \\
\hline & & L mid/post-MTG & $21 / 22$ & & & 4.93 & -62 & -34 & 0 \\
\hline & & LIFG & & 1005 & $<.001$ & & & & \\
\hline & & L post-IFG(Oper) & 44 & & & 4.54 & -46 & 12 & 20 \\
\hline & & L ant-IFG(Orb) & 47 & & & 4.50 & -38 & 36 & -20 \\
\hline & & L IFG(Tri) & 45 & & & 4.46 & -54 & 28 & 2 \\
\hline & & $R$ anterior $M / I T G$ & & 679 & $<.001$ & & & & \\
\hline & & $\mathrm{R}$ ant-M/ITG & $20 / 21$ & & & 5.99 & 52 & -6 & -26 \\
\hline & & $\mathrm{R}$ ant-MTG/TempPole & 21 & & & 5.43 & 62 & 4 & -18 \\
\hline & & $\mathrm{R}$ mid-M/ITG & $20 / 21$ & & & 4.87 & 50 & -16 & -20 \\
\hline & & LpITG/LFuG & & 573 & .001 & & & & \\
\hline & & L post-ITG/IOcG/FuG & 37 & & & 4.79 & -44 & -62 & -10 \\
\hline & & L FuG & 37 & & & 4.51 & -42 & -56 & -20 \\
\hline & & L FuG & $19 / 37$ & & & 4.19 & -40 & -64 & -18 \\
\hline & & ROcG & & 439 & .006 & & & & \\
\hline & & R IOcG & 19 & & & 4.24 & 42 & -82 & -10 \\
\hline & & R IOcG & $18 / 19$ & & & 4.20 & 36 & -92 & -4 \\
\hline & & R OcG/post-ITG & 19 & & & 3.77 & 52 & -78 & -2 \\
\hline & $\mathrm{WA}>\mathrm{WU}$ & $L \mathrm{mid} /$ post-M/STG & & 382 & .013 & & & & \\
\hline & & L post-M/STG & $22 / 42$ & & & 4.25 & -56 & -38 & 12 \\
\hline & & L mid-MTG & 21 & & & 4.00 & -60 & -28 & -4 \\
\hline & & L mid-M/STG & $21 / 22$ & & & 3.28 & -56 & -30 & 4 \\
\hline LaIFG & $\mathrm{SA}>\mathrm{SU}$ & No significant clusters & & & & & & & \\
\hline$\left[\begin{array}{lll}-42 & 26 & 6\end{array}\right]$ & $\mathrm{WA}>\mathrm{WU}$ & No significant clusters & & & & & & & \\
\hline BA $45 / 47$ & & & & & & & & & \\
\hline
\end{tabular}

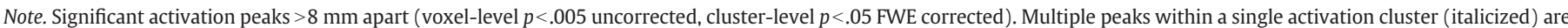

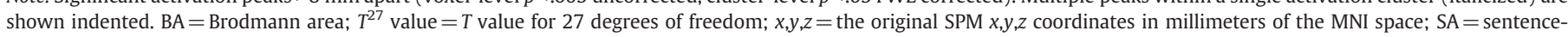

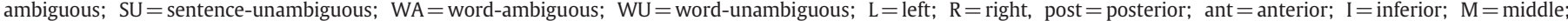

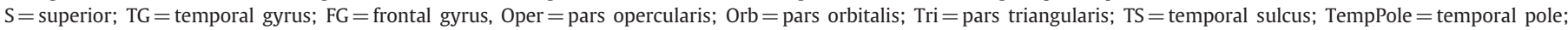
$\mathrm{FuG}=$ fusiform gyrus; OcG = occipital gyrus.

and LPMTGi are modulating the activity of a region that is situated slightly posterior to our ROI in LpMTGi (see Fig. 3A). Fig. 3B displays the time-course of LpMTGi and the more superior LpMTGs for SA, SU, WA, and WU conditions. An ANOVA on the mean contrast estimates of LpMTGi and LpMTGs showed that LpMTGs was activated more strongly than LPMTGi for both word sequence and sentence conditions ( $F_{\text {region }}(1,27)=31.71, p<.001$, see Fig. 3B). Furthermore, the grammaticality effect (sentences $>$ word sequences) was larger in LpMTGs $\left(F_{\text {region } \times \text { gram }}(1,27)=11.36, p=.002\right.$; $F_{\text {LpMTGi: } \operatorname{gram}}(1,27)=50.72 ; p<.001 ; F_{\text {LpMTGs: }}$ gram $(1,27)=111.97$; $p<.001)$, while the main effect of ambiguity was only significant in LpMTGi $\left(F_{\text {region } \times \text { amb }}(1,27)=8.65, p=.007 ; F_{\text {LpMTGi: amb }}(1,27)\right.$ $=13.38 ; p=.001 ; F_{\text {LpMTGs: }}$ amb $\left.(1,27)=1.20 ; p=.283\right)$. For both regions there was an ambiguity by grammaticality interaction ( $F$ region $\times$ amb $\times$ gram $<1 ; F_{\text {LpMTGi: } \text { amb } \times \text { gram }}=6.98, p=.014 ; F_{\text {LpMTGs: }}$ amb $\times^{-}$ gram $=7.47, p=.011)$, with a significant effect of ambiguity in the sentence condition (SA $>$ SU: LpMTGi: $T(27)=4.72, p<.001$; LpMTGs: $T$ $(27)=2.53, p=.009$; WA $>$ WU: LpMTGi: $T(27)=1.40, p=.086$; LpMTGs: $T(27)=-1.18, p=.125)$.

\section{Right hemisphere}

\section{Seed region: RIFG}

The RIFG showed more connectivity for sentence-ambiguous compared to sentence-unambiguous conditions with the precuneus, LpMTGs, RpMTGs, and R anterior/mid-MTG (see Table 3 and Fig. 4A).
Note that the coupling between RIFG and LIFG was not modulated by sentence ambiguity. RIFG did not show larger connectivity to other brain regions for word-ambiguous compared to word-unambiguous conditions.

\section{Seed region: RpMTG}

RpMTG showed enhanced coupling for sentence-ambiguous compared to sentence-unambiguous conditions with LMTG, LIFG, the left fusiform gyrus (LFuG, VWFA), and R mid-MTG (see Table 3 and Fig. 4B). RpMTG showed no larger connectivity with other brain regions for word-ambiguous compared to word-unambiguous conditions.

\section{Subcortical regions}

\section{Seed regions: striatum}

The PPI results for the $L+R$ striatum can be seen in Table 4 and Fig. 5. Both right and left striata showed enhanced coupling with several brain regions for ambiguous compared to unambiguous conditions.

For sentence-ambiguous compared to sentence-unambiguous conditions Rstriatum showed a larger connectivity with LpMTGs and ROcG, while the enhanced coupling with LIFG did not survive multiple comparisons correction. Lstriatum showed a larger connectivity with LPMTGs, RMTG, and LPIFG for sentence-ambiguous compared to sentence-unambiguous conditions. For word-ambiguous compared to word-unambiguous conditions, connectivity was 
Table 3

PPI connectivity results for right hemispheric cortical seed regions (RIFG and RpMTG).

\begin{tabular}{|c|c|c|c|c|c|c|c|c|c|}
\hline Seed & PPI & Region & $\mathrm{BA}$ & $\begin{array}{l}\text { Cluster } \\
\text { size }\end{array}$ & $\begin{array}{l}\text { Cluster } p \\
\text { (corrected) }\end{array}$ & $\begin{array}{l}\text { Voxel } \\
T^{27} \text { value }\end{array}$ & $x$ & $y$ & $z$ \\
\hline RIFG & $\mathrm{SA}>\mathrm{SU}$ & Calcarine gyrus/Pcun & & 1299 & $<.001$ & & & & \\
\hline$\left[\begin{array}{llll}46 & 28 & 6\end{array}\right]$ & & L CalcG & $17 / 18$ & & & 4.79 & -12 & -70 & 12 \\
\hline \multirow[t]{15}{*}{ BA 45} & & Lingual G/CalcG & 17 & & & 4.76 & 0 & -64 & 10 \\
\hline & & Pcun/CalcG & $30 / 17 / 23$ & & & 4.00 & -4 & -56 & 12 \\
\hline & & $L$ posterior M/STG & & 853 & $<.001$ & & & & \\
\hline & & L post-MTG & $39 / 21 / 37$ & & & 4.37 & -50 & -60 & 20 \\
\hline & & L post-MTG/STS & $21 / 22$ & & & 4.01 & -52 & -50 & 8 \\
\hline & & L post-MTG/STS & $21 / 37$ & & & 3.98 & -56 & -54 & 14 \\
\hline & & $R$ posterior $M / S T G$ & & 541 & .001 & & & & \\
\hline & & R post-M/STG & 39 & & & 4.88 & 58 & -66 & 22 \\
\hline & & R post-MTG & 37 & & & 4.67 & 58 & -62 & 10 \\
\hline & & R post-MTG/AngG & 39 & & & 3.46 & 52 & -72 & 26 \\
\hline & & $R$ anterior/mid-MTG & & 346 & .018 & & & & \\
\hline & & $\mathrm{R}$ ant-MTG & 21 & & & 3.98 & 52 & -2 & -24 \\
\hline & & R mid-MTG & $20 / 21$ & & & 3.75 & 68 & -22 & -18 \\
\hline & & $\mathrm{R}$ ant-ITG & $20 / 21$ & & & 3.72 & 58 & -6 & -30 \\
\hline & $W A>W U$ & No significant clusters & & & & & & & \\
\hline RpMTG & $\mathrm{SA}>\mathrm{SU}$ & LMTG & & 2232 & $<.001$ & & & & \\
\hline$[48-34-14]$ & & L mid-MTG & 20 & & & 5.57 & -50 & -20 & -16 \\
\hline \multirow[t]{15}{*}{ BA 20} & & L mid/post-MTG & 21 & & & 5.47 & -56 & -32 & -6 \\
\hline & & L post-MTG & $21 / 37$ & & & 4.96 & -48 & -48 & 2 \\
\hline & & LIFG & & 1017 & $<.001$ & & & & \\
\hline & & L post-IFG(Tri/Oper) & $44 / 45$ & & & 4.80 & -44 & 22 & 18 \\
\hline & & L post-IFG/FOp & $44 / 45$ & & & 3.96 & -36 & 18 & 18 \\
\hline & & L ant-IFG(Orb) & 47 & & & 3.82 & -42 & 36 & -24 \\
\hline & & LFuG & & 626 & $<.001$ & & & & \\
\hline & & L FuG/L ITG & 37 & & & 4.82 & -46 & -60 & -18 \\
\hline & & L IOcG & $37 / 19$ & & & 4.32 & -48 & -68 & -14 \\
\hline & & L FuG & 37 & & & 3.90 & -34 & -56 & -16 \\
\hline & & $R$ mid-MTG & & 599 & $<.001$ & & & & \\
\hline & & R mid-MTG & $20 / 21$ & & & 5.18 & 56 & -22 & -14 \\
\hline & & R mid-MTG & $20 / 21$ & & & 4.22 & 48 & -26 & -10 \\
\hline & & R mid-MTG & $21 / 20 / 37$ & & & 3.98 & 48 & -34 & -8 \\
\hline & $\mathrm{WA}>\mathrm{WU}$ & No significant clusters & & & & & & & \\
\hline
\end{tabular}

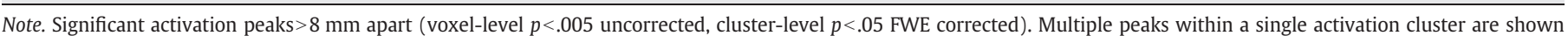
indented. Pcun = precuneus; CalcG = calcarine gyrus; AngG = angular gyrus; FOp = frontal operculum; for additional abbreviations see Table 2.

Table 4

PPI connectivity results for subcortical seed regions (Rstriatum and Lstriatum).

\begin{tabular}{|c|c|c|c|c|c|c|c|c|c|}
\hline & PPI & Region & $\mathrm{BA}$ & $\begin{array}{l}\text { Cluster } \\
\text { size }\end{array}$ & $\begin{array}{l}\text { Cluster } p \\
\text { (corrected) }\end{array}$ & $\begin{array}{l}\text { Voxel } \\
T^{27} \text { value }\end{array}$ & $x$ & $y$ & $z$ \\
\hline \multirow{9}{*}{$\begin{array}{l}\text { R striatum } \\
{\left[\begin{array}{lll}24 & 0 & 8\end{array}\right]}\end{array}$} & $\mathrm{SA}>\mathrm{SU}$ & LpMTG/STS & & 910 & $<.001$ & & & & \\
\hline & & L post-STS & $39 / 21$ & & & 5.31 & -40 & -50 & 16 \\
\hline & & L post-STS & 21 & & & 4.31 & -44 & -44 & 8 \\
\hline & & L post-MTG & $37 / 21$ & & & 4.18 & -44 & -52 & 0 \\
\hline & & $R O c G$ & & 468 & .001 & & & & \\
\hline & & R IOcG & 18 & & & 4.67 & 30 & -94 & -4 \\
\hline & & R IOcG & 19 & & & 4.37 & 44 & -86 & -10 \\
\hline & & R IOCG/lingual G & 18 & & & 4.35 & 28 & -90 & -12 \\
\hline & $\mathrm{WA}>\mathrm{WU}$ & No significant clusters & & & & & & & \\
\hline \multirow{15}{*}{$\begin{array}{l}\text { L striatum } \\
{\left[\begin{array}{lll}-24 & 0 & 8\end{array}\right]}\end{array}$} & $\mathrm{SA}>\mathrm{SU}$ & L posterior MTG & & 1244 & $<.001$ & & & & \\
\hline & & L post-MTG & $21 / 37$ & & & 5.38 & -54 & -52 & 0 \\
\hline & & L post-MTG & $21 / 37$ & & & 5.14 & -46 & -50 & 2 \\
\hline & & L post-MTG/STS & 21 & & & 4.57 & -50 & -52 & 12 \\
\hline & & $R$ mid-posterior MTG & & 417 & .005 & & & & \\
\hline & & R mid-MTG & $20 / 21$ & & & 4.77 & 50 & -22 & -10 \\
\hline & & R mid-M/ITG & $20 / 37$ & & & 4.27 & 46 & -30 & -14 \\
\hline & & R post-MTG & 21 & & & 3.76 & 50 & -46 & -2 \\
\hline & & $L$ posterior IFG & & 337 & .015 & & & & \\
\hline & & L post-IFG(Oper) & 44 & & & 4.72 & -38 & 10 & 28 \\
\hline & & L post-IFG(Oper/Tri) & 44 & & & 3.93 & -46 & 12 & 28 \\
\hline & $\mathrm{WA}>\mathrm{WU}$ & $L$ OcG/L posterior ITG & & 627 & .001 & & & & \\
\hline & & L IOcG & $18 / 19$ & & & 4.36 & -38 & -88 & -10 \\
\hline & & L IOcG/FuG & 19 & & & 4.34 & -44 & -82 & -12 \\
\hline & & L FuG/post-ITG & $37 / 19$ & & & 4.23 & -42 & -62 & -18 \\
\hline
\end{tabular}

Note. Significant clusters (voxel-level $p<.005$ uncorrected, cluster-level $p<.05$ FWE corrected). For abbreviations see Table 2. 
A Seed RIFG; PPI SA $>$ SU

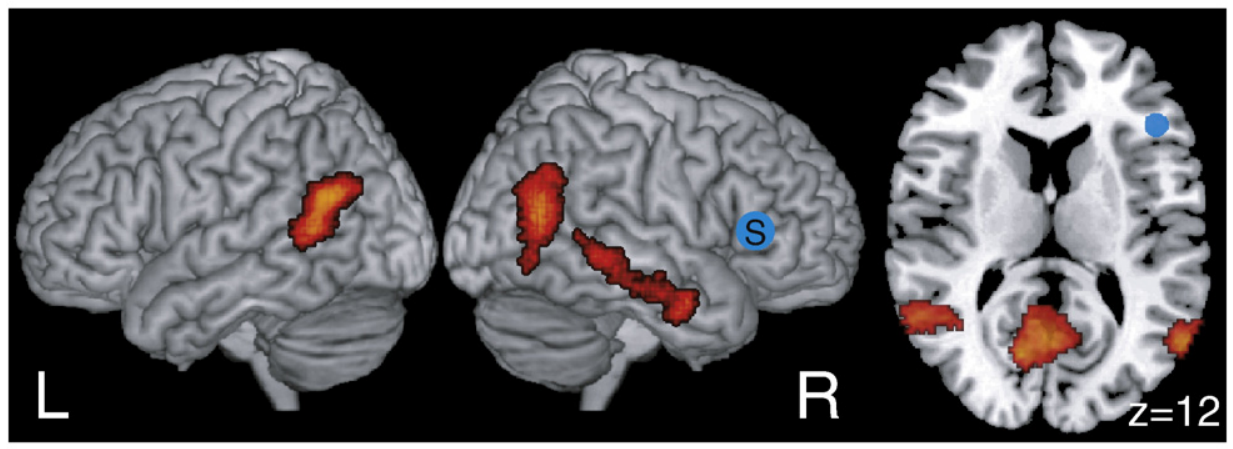

B Seed RpMTG; PPI SA>SU

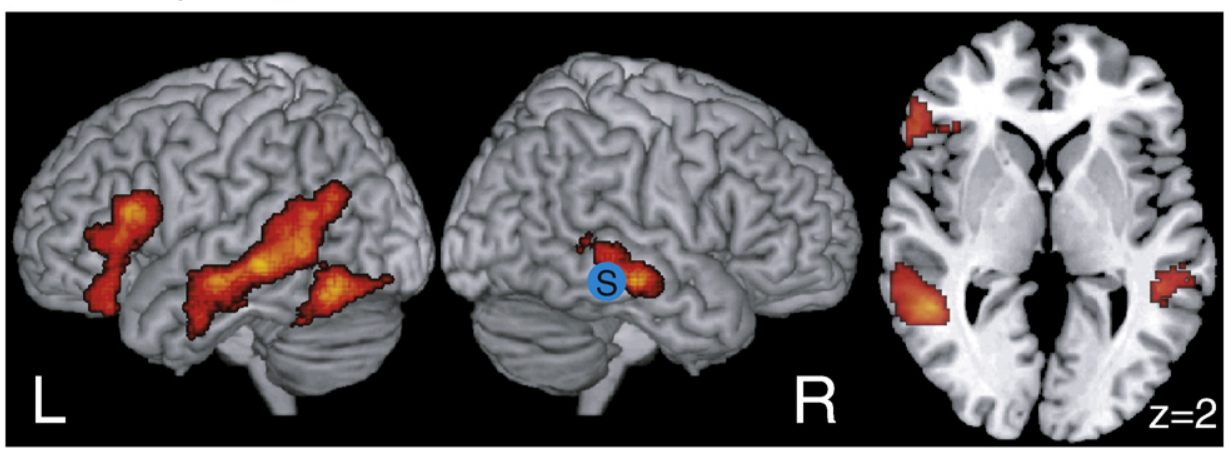

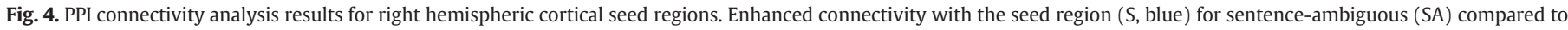

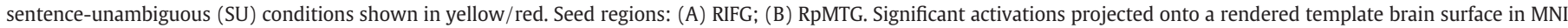

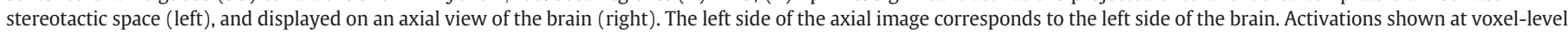
$P_{\text {uncorr }}<.005$, cluster-level $P_{\text {FWE }}<.05$.

significantly enhanced between Lstriatum and LOcG/LpITG only (see Table 4 and Fig. 5A).

\section{Discussion}

The main goal of the current study was to identify whether unification processes in language modulate the connectivity between left frontal and temporal brain regions. We hypothesized that in word-category ambiguous sentences the effective connectivity between LIFG and LPMTG would be larger than in unambiguous sentences, while this ambiguity effect would not be present in word sequences. Our results showed that this was the case. For sentence ambiguous compared to sentence unambiguous conditions, LpIFG showed larger connectivity to LpMTGs, while LpMTGi showed larger connectivity to LIFG (and several other regions in the sentence processing network). No such enhanced coupling with ambiguity was observed between LIFG and LMTG in the context of word sequences. Thus, our results support the hypothesis of enhanced connectivity between left frontal and temporal regions during the unification process. However, we did not see direct effective connectivity between LPIFG and LpMTGi; instead both regions were modulating a separate brain region slightly superior to LpMTGi: LpMTGs (see Fig. 3). As Fig. 3B shows, LpMTGs was highly activated for words and even more so for sentences. Interestingly, in addition to the seed regions in LpIFG and LPMTGi, LpMTGs also showed enhanced coupling with the seed regions in RIFG, RpMTG, and the striatum for sentence ambiguous compared to sentence unambiguous words (see Figs. 4-5 and Tables 2-4). Therefore, this region seems to serve as a hub in the language processing network.
The roles of LPMTGi and LPMTGs

Both LpMTGi and LpMTGs are known to be involved in the activation of meanings of words stored in long-term memory ${ }^{1}$ (e.g. Vandenberghe et al., 1996; Gold and Buckner, 2002; Bedny et al., 2008a). Frequently studies have found LPMTGs involvement when the preceding sentence or discourse context results in increased semantic unification load of a word (e.g. Ni et al., 2000; Kuperberg et al., 2003; Xu et al., 2005; Willems et al., 2007; Ferstl et al., 2008; Willems et al., 2008; see Hagoort et al. (2009) for a review). This LpMTGs involvement in semantic unification might be the result of the conceptual representation of the individual words in LpMTGs being constantly maintained and updated by LIFG when new words come in (see also Humphries et al., 2007). While LpMTGi has been implicated in the processing of (semantic) ambiguity in sentences (Rodd et al., 2005; Davis et al., 2007; Zempleni et al., 2007), LpMTGs seems to be especially involved when different sources of information converge on a common conceptual memory representation (Beauchamp et al., 2004; Hein et al., 2007; Hagoort et al., 2009; Willems et al., 2009).

Furthermore, LpMTGs is involved in the processing of syntactically complex sentences, which has been attributed to the integration of lexical-semantic and syntactic information during sentence comprehension (Friederici et al., 2009). Note that here also different sources of information converge on a common memory representation.

Both LPMTGi and LpMTGs are activated more for verbs than for nouns (both: (Grossman et al., 2002; Davis et al., 2004; Bedny et al., 2008b), LpMTGi: (Warburton et al., 1996), LpMTGs: (Shapiro et al., 2006)) and more for mental verbs than for motion verbs (Grossman et al., 2002). Furthermore, LpMTGi is activated more for verbs with 


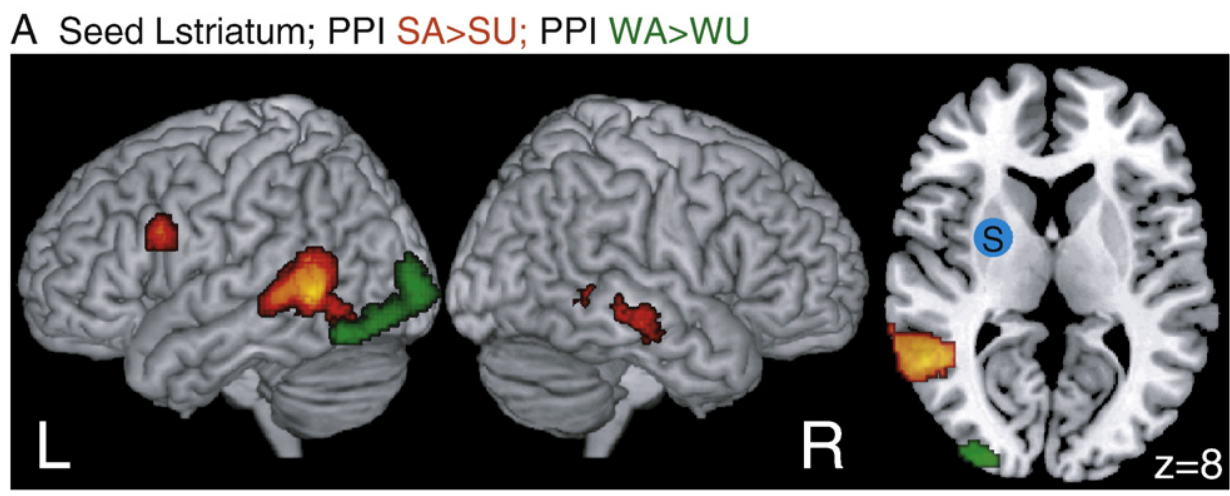

\section{B Seed Rstriatum; PPI SA>SU}

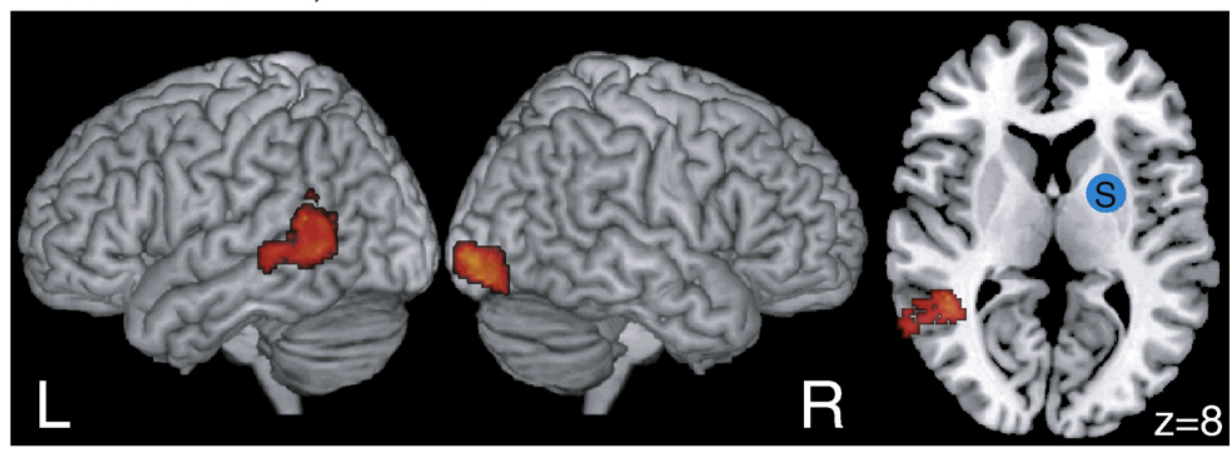

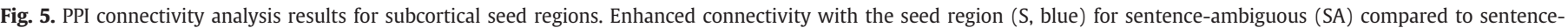

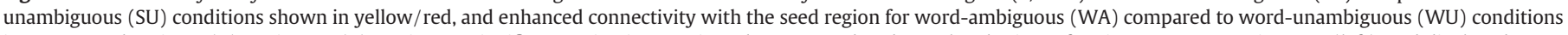

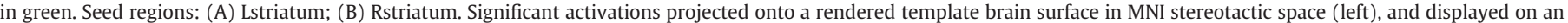
axial view of the brain (right). The left side of the axial image corresponds to the left side of the brain. Activations shown at voxel-level $P_{\text {uncorr }}<.005$, cluster-level $P_{\mathrm{FWE}}<.05$.

multiple verb arguments (den Ouden et al., 2009). This verb effect might be related to either the more difficult semantic (event) structure or the more complex lexical-syntactic information of (mental) verbs (Bedny et al., 2008b). For example, mental verbs such as believe often carry embedded S-nodes ( $X$ believes that $Y$ ), and are thereby syntactically more complex than e.g. action verbs. We hypothesize that LpMTGi activation reflects the retrieval of lexicalsyntactic frames associated with different word categories (noun, verb, determiners, etc.). Results of Davis et al. indicate that in LpMTGs the syntactic word-class effect is presumably more dependent on the semantic rather than syntactic properties of the experimental items (Davis et al., 2004). LpMTGi and LpMTGs might cooperate for retrieval of lexical-syntactic information and activation of appropriate word meanings.

\section{The left hemisphere unification network}

Our interpretation of the present PPI results is as follows: when a word-category ambiguous word is presented, lexical-syntactic information (syntactic templates for noun and verb) is retrieved from memory. This process is subserved by LpMTGi (Snijders et al., 2009). LPMTGi and LpMTGs work in concert for activating the conceptual representation of the word (both in sentence and word sequence context). If the ambiguous word is presented in a sentence context, the lexical-syntactic information given by LPMTGi is used by LpIFG for syntactic unification. During this process, the conceptual representation in LpMTGs is updated and maintained by feedback from inferior frontal gyrus. Future studies will need to give evidence about the precise division of labor for LpMTGi and LpMTGs between activating lexical-syntactic versus conceptual representations.

Both LIFG and LpMTG activation has been described in response to syntactically complex sentences (Keller et al., 2001; Fiebach et al.,
2005), even if stimuli were matched for lexical content (Peelle et al., 2010). One possible interpretation of this pattern of results is that LPMTG plays a role in unification that is similar to that of LIFG. However, we favor an alternative interpretation: Sentence processing requires sustained activation of lexical-syntactic information (Snijders et al., 2009). The lexical-syntactic information is most likely not 'copied' from the area necessary for its retrieval (LPMTG) to the area necessary for unification (LIFG). Instead, the sustained activation of lexical-syntactic information could be triggered by feedback from the LIFG to the LPMTG (see Vosse and Kempen (2009) for a computational implementation hereof). The amount and/or duration of lexicalsyntactic activation is a function of the unification load imposed by the combinatorial operations necessary for unification. Although we suggest a certain division of labor between LpMTG and LIFG in the retrieval and unification of lexical-syntactic information respectively, we hypothesized that the continuous interplay between these regions is needed for successful syntactic unification (Snijders et al., 2009). The current study found direct evidence for LIFG and LPMTG working in concert during syntactic unification processes.

\section{Right hemisphere and the unification network}

Second, in an exploratory fashion, we investigated how RIFG and RpMTG are functionally connected to the unification network. Both $\mathrm{RH}$ regions get involved in sentence processing when confronted with a word-category ambiguity. For word-ambiguous compared to wordunambiguous conditions RIFG and RpMTG did not show enhanced connectivity to other brain regions. For sentence-ambiguous compared to sentence-unambiguous conditions, RpMTG showed differential connectivity to the left hemispheric language processing network. RIFG modulated the response of RpMTGs to sentence ambiguity, in a similar way as LpIFG modulated the response of 
LpMTGs. This suggests that, at least for RIFG, involvement of right hemisphere regions in the unification process is not solely due to interhemispheric connections between homotopic regions. The results are in agreement with data suggesting that the right hemisphere gets involved with sentence processing when context is needed for the disambiguation of alternative interpretations (see e.g. Faust and Chiarello, 1998; Kircher et al., 2001; Bookheimer, 2002; Grindrod and Baum, 2005; Menenti et al., 2009; Tesink et al., 2009).

\section{Striatum and the unification network}

Third, we investigated how the striatum is functionally connected to the unification network. Does the striatum show enhanced connectivity to LIFG and/or LpMTG during ambiguity processing, and does this enhanced connectivity differ in a sentence versus a word-list context? Although in the subtraction analysis there was no difference in striatal ambiguity effect for sentence and word sequence conditions (Snijders et al., 2009), the connectivity analysis showed very different results for the striatum in the context of sentences versus words. For word-ambiguous compared to word-unambiguous conditions the striatum modulated activity in low-level visual regions only. However, for sentence-ambiguous compared to sentenceunambiguous conditions also regions in left and right MTG and in LIFG showed enhanced coupling with the striatum (see Table 4 and Fig. 5). Thus, the PPI analysis revealed that the striatum is functionally connected to cortical regions for word and sentence-level ambiguities, but, importantly, to a different network of cortical regions depending on whether the context is a sentence or a sequence of words.

Syntactic unification requires the maintenance and on-line integration of lexical-syntactic representations over time, and the flexible and selective updating of these representations (selecting among competing alternatives, see Hagoort (2005)). In the context of working memory (WM) research, the focus has traditionally been on the role of the prefrontal cortex (PFC) in maintenance and updating of information. Recently also the striatum has been found to play a role in the updating of representations (Frank et al., 2001; Gruber et al., 2006; Cools, 2008; McNab and Klingberg, 2008). The basal ganglia (BG) might provide a dynamic gating mechanism for WM by momentarily inhibiting or disinhibiting the PFC, thus enabling (but not directly causing) e.g. lexical-syntactic information to be actively relayed to PFC (Hazy et al., 2007). This is much like the BG involvement in gating the selection of actions in motor operations (Mink, 1996) and in agreement with interpretations of BG contribution to language processing (e.g., Crosson et al., 2007).

How can we explain our results in the light of existing knowledge on the basal ganglia? Salient events (such as in our case ambiguous words) elicit dopamine release (Schultz et al., 1993; Zink et al., 2006), thereby reducing the BG threshold for facilitating/suppressing a cortical command in response to particular stimuli (Frank, 2005), and thus providing a relevance signal for cortical areas involved in word processing and unification. In word sequences, the saliency of ambiguous lexical-syntactic representations is only relevant for lower-level word processing regions, which results in higher connectivity of the striatum to LPITG/LOcG (coinciding with the visual word form area (McCandliss et al., 2003)). The unification operations on the lexical-syntactic representations are essential only in a sentence context. Thus, inferior frontal and striatal regions may cooperate for performing unification operations on lexical-syntactic representations retrieved from LpMTG only in this type of context. Evidence that the striatum can modulate information transfer between cortical regions comes from a recent study using dynamic causal modeling (den Ouden et al., 2010).

Obviously, cortico-striatal loops function as an integrated system, where it is difficult to segregate functional roles of nodes in the system. Whether during sentence unification the striatum is directly or indirectly modulating activity in LPIFG, LPMTG, or the connection between these regions, remains a question for further research.

\section{Conclusion: the unification network}

In this study we investigated the unification brain network using psychophysiological interactions. The results show that bilateral inferior frontal and posterior temporal gyri are functionally connected during unification. Furthermore, the striatum is functionally connected to the cortical unification network. Connections between LIFG and the striatum might control the extraction of lexical-syntactic information from left posterior temporal regions in the service of the unification operations subserved by LIFG (see also O'Reilly and Frank 2006; Dayan, 2008). RIFG and RpMTG get involved in unification when context is needed for the disambiguation of alternative interpretations.

Although language in the brain has been studied since the 19th century, the study on network interactions during sentence comprehension has only just begun (Hampson et al., 2002; Homae et al., 2003; Prat et al., 2007; Chow et al., 2008; Tyler and Marslen-Wilson, 2008). The current study adds important insights to this emerging field, and provides important input for further studies on unification dynamics, for example using dynamic causal modeling (Friston et al., 2003).

\section{Acknowledgments}

This research was funded by a grant from the Netherlands Organization for Scientific Research (grant 051.04.030). We thank Roshan Cools, Branka Milivojevic, Jos van Berkum, Gerard Kempen, Ingrid Nieuwenhuis and Miriam Kos for the discussion and comments.

\section{Appendix A. Supplementary data}

Supplementary data associated with this article can be found, in the online version, at doi:10.1016/j.neuroimage.2010.05.035.

\section{References}

Baayen, R.H., Piepenbrock, R., Rijn, H.v., 1993. The CELEX lexical database [CD-ROM]. In. Philadelphia: Linguistic Data Consortium, University of Pennsylvania.

Beauchamp, M.S., Lee, K.E., Argall, B.D., Martin, A., 2004. Integration of auditory and visual information about objects in superior temporal sulcus. Neuron 41, 809-823.

Bedny, M., McGill, M., Thompson-Schill, S.L., 2008a. Semantic adaptation and competition during word comprehension. Cereb. Cortex 18, 2574-2585.

Bedny, M., Caramazza, A., Grossman, E., Pascual-Leone, A., Saxe, R., 2008b. Concepts are more than percepts: the case of action verbs. J. Neurosci. 28, 11347-11353.

Beek, L.v.d., Bouma, G., Malouf, R., Noord, G.v., 2001. The Alpino Dependency Treebank Computational Linguistics in the Netherlands (CLIN). Enschede.

Bookheimer, S., 2002. Functional MRI of language: new approaches to understanding the cortical organization of semantic processing. Annu. Rev. Neurosci. 25, 151-188.

Chomsky, N., 1995. The Minimalist Program. MIT Press, Cambridge, MA.

Chow, H.M., Kaup, B., Raabe, M., Greenlee, M.W., 2008. Evidence of fronto-temporal interactions for strategic inference processes during language comprehension. NeuroImage 40, 940-954.

Cools, R., 2008. Role of dopamine in the motivational and cognitive control of behavior. Neuroscientist 14, 381-395.

Copland, D.A., Chenery, H.J., Murdoch, B.E., 2000. Understanding ambiguous words in biased sentences: evidence of transient contextual effects in individuals with nonthalamic subcortical lesions and Parkinson's disease. Cortex 36, 601-622.

Crosson, B., Benjamin, M., Levy, I., 2007. Role of the basal ganglia in language and semantics: supporting cast. In: Hart, J., Kraut, M.A. (Eds.), Neural Basis of Semantic Memory. Cambridge University Press, Cambridge, pp. 219-243.

Curtis, C.E., D'Esposito, M., 2003. Persistent activity in the prefrontal cortex during working memory. Trends Cogn. Sci. 7, 415-423.

Davis, M.H., Meunier, F., Marslen-Wilson, W.D., 2004. Neural responses to morphological, syntactic, and semantic properties of single words: an fMRI study. Brain Lang 89, 439-449.

Davis, M.H., Coleman, M.R., Absalom, A.R., Rodd, J.M., Johnsrude, I.S., Matta, B.F., Owen, A.M., Menon, D.K., 2007. Dissociating speech perception and comprehension at reduced levels of awareness. Proc. Natl. Acad. Sci. U. S. A. 104, 16032-16037.

Dayan, P., 2008. Simple substrates for complex cognition. Front. Neurosci. 2, 255-264. 
den Ouden, D.B., Fix, S., Parrish, T.B., Thompson, C.K., 2009. Argument structure effects in action verb naming in static and dynamic conditions. J. Neurolinguistics 22 , 196-215.

den Ouden, H.E.M., Daunizeau, J., Roiser, J.P., Friston, K.J., Stephan, K.E., 2010. Striatal prediction error modulates cortical coupling. J. Neurosci. 30, 3210-3219.

Duffy, S.A., Morris, R.K., Rayner, K., 1988. Lexical ambiguity and fixation times in reading. J. Mem. Lang. 27, 429-446.

Evans, A.C., Collins, D.L., Mills, S.R., Brown, E.D., Kelly, R.L., Peters, T.M., 1993. 3D statistical neuroanatomical models from 305 MRI volumes. IEEE-Nuclear Science Symposium and Medical Imaging Conference, pp. 1813-1817.

Faust, M., Chiarello, C., 1998. Sentence context and lexical ambiguity resolution by the two hemispheres. Neuropsychologia 36, 827-835.

Ferstl, E.C., Neumann, J., Bogler, C., von Cramon, D.Y., 2008. The extended language network: a meta-analysis of neuroimaging studies on text comprehension. Hum. Brain Mapp. 29, 581-593.

Fiebach, C.J., Schlesewsky, M., Lohmann, G., von Cramon, D.Y., Friederici, A.D., 2005. Revisiting the role of Broca's area in sentence processing: syntactic integration versus syntactic working memory. Hum. Brain Mapp. 24, 79-91.

Fiebach, C.J., Rissman, J., D'Esposito, M., 2006. Modulation of inferotemporal cortex activation during verbal working memory maintenance. Neuron 51, 251-261.

Forman, S.D., Cohen, J.D., Fitzgerald, M., Eddy, W.F., Mintun, M.A., Noll, D.C., 1995 Improved assessment of significant activation in functional magnetic-resonanceimaging (fMRI) - use of a cluster-size threshold. Magn. Reson. Med. 33, 636-647.

Frank, M.J., 2005. Dynamic dopamine modulation in the basal ganglia: a neurocomputational account of cognitive deficits in medicated and nonmedicated Parkinsonism. J. Cogn. Neurosci. 17, 51-72.

Frank, M.J., Loughry, B., O'Reilly, R.C., 2001. Interactions between the frontal cortex and basal ganglia in working memory: a computational model. Cogn. Affect. Behav. Neurosci. 1, 137-160.

Friederici, A.D., Ruschemeyer, S.A., Hahne, A., Fiebach, C.J., 2003. The role of left inferior frontal and superior temporal cortex in sentence comprehension: localizing syntactic and semantic processes. Cereb. Cortex 13, 170-177.

Friederici, A.D., Makuuchi, M., Bahlmann, J., 2009. The role of the posterior superior temporal cortex in sentence comprehension. NeuroReport 20, 563-568.

Frisch, S., Kotz, S.A., von Cramon, D.Y., Friederici, A.D., 2003. Why the P600 is not just a P300: the role of the basal ganglia. Clin. Neurophysiol. 114, 336-340.

Friston, K.J., 2002. Beyond phrenology: what can neuroimaging tell us about distributed circuitry? Annu. Rev. Neurosci. 25, 221-250.

Friston, K.J., Holmes, A., Poline, J.B., Price, C.J., Frith, C.D., 1996. Detecting activations in PET and fMRI: levels of inference and power. Neurolmage 4, 223-235.

Friston, K.J., Buechel, C., Fink, G.R., Morris, J., Rolls, E., Dolan, R.J., 1997. Psychophysiological and modulatory interactions in neuroimaging. Neurolmage 6, 218-229.

Friston, K.J., Harrison, L., Penny, W., 2003. Dynamic causal modelling. NeuroImage 19, 1273-1302.

Friston, K.J., Ashburner, J.T., Kiebel, S.J., Nichols, T.E., Penny, W.D., 2007. Statistical Parametric Mapping: the Analysis of Functional Brain Images. Academic Press, San Diego, CA.

Fuster, J.M., 2001. The prefrontal cortex - an update: time is of the essence. Neuron 30, 319-333.

Fuster, J.M., 2008. The Prefrontal Cortex, 4th ed. Academic Press, London.

Gitelman, D.R., Penny, W.D., Ashburner, J., Friston, K.J., 2003. Modeling regional and psychophysiologic interactions in fMRI: the importance of hemodynamic deconvolution. NeuroImage 19, 200-207.

Gold, B.T., Buckner, R.L., 2002. Common prefrontal regions coactivate with dissociable posterior regions during controlled semantic and phonological tasks. Neuron 35 , 803-812.

Grindrod, C.M. Baum, S.R, 2005. Hemispheric contributions to lexical ambiguity resolution in a discourse context: evidence from individuals with unilateral left and right hemisphere lesions. Brain Cogn. 57, 70-83.

Grossman, M., Koenig, P., DeVita, C., Glosser, G., Alsop, D., Detre, J., Gee, J., 2002. Neura representation of verb meaning: an fMRI study. Hum. Brain Mapp. 15, 124-134.

Gruber, A.J., Dayan, P., Gutkin, B.S., Solla, S.A., 2006. Dopamine modulation in the basal ganglia locks the gate to working memory. J. Comput. Neurosci. 20, 153-166.

Hagoort, P., 2005. On Broca, brain, and binding: a new framework. Trends Cogn. Sci. 9, 416-423.

Hagoort, P., Baggio, G., Willems, R.M., 2009. Semantic unification. In: Gazzaniga, M.S. (Ed.), The Cognitive Neurosciences IV. MIT Press, Cambridge, MA, pp. 819-836.

Hampson, M., Peterson, B.S., Skudlarski, P., Gatenby, J.C., Gore, J.C., 2002. Detection of functional connectivity using temporal correlations in MR images. Hum. Brain Mapp. 15, 247-262.

Hazy, T.E., Frank, M.J., O'Reilly, R.C., 2007. Towards an executive without a homunculus: computational models of the prefrontal cortex/basal ganglia system. Philos. Trans. R. Soc. B Biol. Sci. 362, 1601-1613.

Hein, G., Knight, R.T., 2008. Superior temporal sulcus-it's my area: or is it? J. Cogn. Neurosci. 20, 2125-2136.

Hein, G., Doehrmann, O., Muller, N.G., Kaiser, J., Muckli, L., Naumer, M.J., 2007. Object familiarity and semantic congruency modulate responses in cortical audiovisual integration areas. J. Neurosci. 27, 7881-7887.

Homae, F., Yahata, N., Sakai, K.L., 2003. Selective enhancement of functional connectivity in the left prefrontal cortex during sentence processing. Neurolmage 20, 578-586

Humphries, C., Binder, J.R., Medler, D.A., Liebenthal, E., 2007. Time course of semantic processes during sentence comprehension: an fMRI study. NeuroImage 36, 924-932.

Jackendoff, R., 2002. Foundations of Language: Brain, Meaning, Grammar, Evolution. Oxford University Press, Oxford.
Jung-Beeman, M., 2005. Bilateral brain processes for comprehending natural language. Trends Cogn. Sci. 9, 512-518.

Keller, T.A., Carpenter, P.A., Just, M.A., 2001. The neural bases of sentence comprehension: a fMRI examination of syntactic and lexical processing. Cereb. Cortex 11, 223-237.

Kircher, T.T.J., Brammer, M., Andreu, N.T., Williams, S.C.R., McGuire, P.K., 2001. Engagement of right temporal cortex during processing of linguistic context. Neuropsychologia 39, 798-809.

Kuperberg, G.R., Holcomb, P.J., Sitnikova, T., Greve, D., Dale, A.M., Caplan, D., 2003. Distinct patterns of neural modulation during the processing of conceptual and syntactic anomalies. J. Cogn. Neurosci. 15, 272-293.

Lancaster, J.L., Woldorff, M.G., Parsons, L.M., Liotti, M., Freitas, E.S., Rainey, L., Kochunov, P.V., Nickerson, D., Mikiten, S.A., Fox, P.T., 2000. Automated Talairach Atlas labels for functional brain mapping. Hum. Brain Mapp. 10, 120-131.

Levelt, W.J.M., 1992. Accessing words in speech production - stages, processes and representations. Cognition 42, 1-22.

Lieberman, P., 2001. Human language and our reptilian brain - the subcortical bases of speech, syntax, and thought. Perspect. Biol. Med. 44, 32-51.

Mai, J.K., Assheuer, J., Paxinos, G., 2004. Atlas of the Human Brain, Second Edition. Elsevier Academic Press, London.

Marslen-Wilson, W.D., 1987. Functional parallelism in spoken word-recognition. Cognition 25, 71-102.

McCandliss, B.D., Cohen, L., Dehaene, S., 2003. The visual word form area: expertise for reading in the fusiform gyrus. Trends Cogn. Sci. 7, 293-299.

McNab, F., Klingberg, T., 2008. Prefrontal cortex and basal ganglia control access to working memory. Nat. Neurosci. 11, 103-107.

Menenti, L., Petersson, K.M., Scheeringa, R., Hagoort, P., 2009. When elephants fly: differential sensitivity of right and left inferior frontal gyri to discourse and world knowledge. J. Cogn. Neurosci. 21, 2358-2368.

Miller, E.K., Cohen, J.D., 2001. An integrative theory of prefrontal cortex function. Annu. Rev. Neurosci. 24, 167-202.

Miller, B.T., D'Esposito, M., 2005. Searching for "the top" in top-down control. Neuron 48, 535-538.

Mink, J.W., 1996. The basal ganglia: focused selection and inhibition of competing motor programs. Prog. Neurobiol. 50, 381-425.

Ni, W., Constable, R.T., Mencl, W.E., Pugh, K.R., Fulbright, R.K., Shaywitz, S.E., Shaywitz, B.A., Gore, J.C., Shankweiler, D., 2000. An event-related neuroimaging study distinguishing form and content in sentence processing. J. Cogn. Neurosci. 12, 120-133.

O'Reilly, R.C., Frank, M.J., 2006. Making working memory work: a computational model of learning in the prefrontal cortex and basal ganglia. Neural Comput. 18, 283-328.

Peelle, J., Troiani, V., Wingfield, A., Grossman, M., 2010. Neural processing during older adults' comprehension of spoken sentences: age differences in resource allocation and connectivity. Cereb. Cortex 20, 773-782.

Petersson, K.M., Forkstam, C., Ingvar, M., 2004. Artificial syntactic violations activate Broca's region. Cogn. Sci. 28, 383-407.

Prat, C.S., Keller, T.A., Just, M.A., 2007. Individual differences in sentence comprehension: a functional magnetic resonance imaging investigation of syntactic and lexical processing demands. J. Cogn. Neurosci. 19, 1950-1963.

Rodd, J.M., Davis, M.H., Johnsrude, I.S., 2005. The neural mechanisms of speech comprehension: fMRI studies of semantic ambiguity. Cereb. Cortex 15, 1261-1269.

Rorden, C., Brett, M., 2000. Stereotaxic display of brain lesions. Behav. Neurol. 12, 191-200.

Schultz, W., Apicella, P., Ljungberg, T., 1993. Responses of monkey dopamine neurons to reward and conditioned-stimuli during successive steps of learning a delayedresponse task. J. Neurosci. 13, 900-913.

Seidenberg, M.S., Tanenhaus, M.K., Leiman, J.M., Bienkowski, M., 1982. Automatic access of the meanings of ambiguous words in context - some limitations of knowledgebased processing. Cogn. Psychol. 14, 489-537.

Shapiro, K.A., Moo, L.R., Caramazza, A., 2006. Cortical signatures of noun and verb production. Proc. Natl. Acad. Sci. U. S. A. 103, 1644-1649.

Snijders, T.M., Vosse, T., Kempen, G., Van Berkum, J.J.A., Petersson, K.M., Hagoort, P., 2009. Retrieval and unification of syntactic structure in sentence comprehension: an fMRI study using word-category ambiguity. Cereb. Cortex 19, 1493-1503.

Tesink, C.M.J.Y., Petersson, K.M., van Berkum, J.J.A., van den Brink, D., Buitelaar, J.K., Hagoort, P., 2009. Unification of speaker and meaning in language comprehension: an fMRI study. J. Cogn. Neurosci. 21, 2085-2099.

Thompson-Schill, S.L., D'Esposito, M., Aguirre, G.K., Farah, M.J., 1997. Role of left inferior prefrontal cortex in retrieval of semantic knowledge: a reevaluation. Proc. Natl. Acad. Sci. U. S. A. 94, 14792-14797.

Tomita, H., Ohbayashi, M., Nakahara, K., Hasegawa, I., Miyashita, Y., 1999. Top-down signal from prefrontal cortex in executive control of memory retrieval. Nature 401, 699-703.

Tyler, L.K., Marslen-Wilson, W., 2008. Fronto-temporal brain systems supporting spoken language comprehension. Philos. Trans. R. Soc. B Biol. Sci. 363, 1037-1054.

Tzourio-Mazoyer, N., Landeau, B., Papathanassiou, D., Crivello, F., Etard, O., Delcroix, N., Mazoyer, B., Joliot, M., 2002. Automated anatomical labeling of activations in SPM using a macroscopic anatomical parcellation of the MNI MRI single-subject brain. NeuroImage 15, 273-289.

Ullman, M.T., 2004. Contributions of memory circuits to language: the declarative/ procedural model. Cognition 92, 231-270.

Vandenberghe, R., Price, C., Wise, R., Josephs, O., Frackowiak, R.S.J., 1996. Functional anatomy of a common semantic system for words and pictures. Nature 383 , 254-256.

Vosse, T., Kempen, G., 2000. Syntactic structure assembly in human parsing: a computational model based on competitive inhibition and a lexicalist grammar. Cognition 75, 105-143. 
Vosse, T., Kempen, G., 2009. The Unification Space implemented as a localist neural net: predictions and error-tolerance in a constraint-based parser. Cogn. Neurodyn. 3, 331-346.

Warburton, E., Wise, R.J.S., Price, C.J., Weiller, C., Hadar, U., Ramsay, S., Frackowiak, R.S.J., 1996. Noun and verb retrieval by normal subjects studies with PET. Brain 119 , 159-179.

Willems, R.M., Ozyurek, A., Hagoort, P., 2007. When language meets action: the neural integration of gesture and speech. Cereb. Cortex 17, 2322-2333.

Willems, R.M., Ozyurek, A., Hagoort, P., 2008. Seeing and hearing meaning: ERP and fMRI evidence of word versus picture integration into a sentence context. J. Cogn. Neurosci. 20, 1235-1249.
Willems, R.M., Ozyurek, A., Hagoort, P., 2009. Differential roles for left inferior frontal and superior temporal cortex in multimodal integration of action and language. NeuroImage 47, 1992-2004.

Xu, J., Kemeny, S., Park, G., Frattali, C., Braun, A., 2005. Language in context: emergent features of word, sentence, and narrative comprehension. Neurolmage 25 1002-1015.

Zempleni, M.Z., Renken, R., Hoeks, J.C.J., Hoogduin, J.M., Stowe, L.A., 2007. Semantic ambiguity processing in sentence context: evidence from event-related fMRI Neurolmage 34, 1270-1279.

Zink, C.F., Pagnoni, G., Chappelow, J., Martin-Skurski, M., Berns, G.S., 2006. Human striatal activation reflects degree of stimulus saliency. NeuroImage 29, 977-983. 